\title{
Influence of ionospheric conductivity on mid-latitude Pc 3-4 pulsations
}

\author{
N. Yagova ${ }^{1}$, V. Pilipenko ${ }^{1}$, E. Fedorov ${ }^{1}$, M. Vellante ${ }^{2}$, and K. Yumoto ${ }^{3}$ \\ ${ }^{1}$ Institute of the Physics of the Earth, Moscow 123810, Russia \\ 2 ''Aquila University, l'Aquila 67010, Italy \\ ${ }^{3}$ Kyushu University 33, Fukuoka 812-8581, Japan
}

(Received November 10, 1997; Revised December 24, 1998; Accepted December 24, 1998)

\begin{abstract}
Diurnal variations of the parameters of the magnetospheric Alfvén resonator at different latitudes have been calculated using a semi-empirical model of the ionosphere-magnetosphere plasma distribution. The ionospheric plasma density is taken from the IRI model, the electron density at the magnetospheric equator is based on the ISEE/whistler model, and the field-aligned magnetospheric plasma distribution is calculated under the assumption of diffusive equilibrium. It is shown that for the mid-latitude ionosphere the Hall conductivity has no effect on the parameters of the magnetospheric Alfvén resonator. The calculated values of damping rates of Alfvén oscillations at middle latitudes during the dark period are too high for the "free-end" and "quarter-wave" oscillation regimes to be realized. At low latitudes quality factors are relatively high both at daytime and nighttime conditions. An expected change of a field-aligned structure of Alfvén oscillations during the transition from dayside to nightside ionospheric conditions does not occur. The analysis of the experimental data recorded at middle and low latitude stations of the " $210^{\circ}$ Magnetic Meridian" magnetometer network and station l'Aquila gives the results, compatible with the predictions of the numerical model: (a) the pulsation amplitude in a frequency band near the fundamental harmonic of the Alfvén field line resonance has the strongest dependence on the ionospheric conductivity; (b) the influence of day/night ionospheric conditions on the Pc 3 amplitudes is less at low $(L \leq 2)$ geomagnetic latitudes than at middle latitudes; (c) the ionospheric conductivity control of the Pc 3 amplitude at middle latitudes weakens with increasing harmonic number.
\end{abstract}

\section{Introduction}

Joule dissipation in the ionosphere is one of the most important mechanisms determining the quality factor $Q$ of the magnetospheric Alfvén resonator. Via this mechanism the ionosphere controls the rate of resonant amplification of incident ULF wave energy at frequencies of local Alfvén eigen oscillations. In the simplest case of a uniform magnetosphere and a thin horizontally-uniform ionosphere, the $Q$-factor depends only on the ratio between the height-integrated Pedersen conductivity of the ionosphere $\Sigma_{\mathrm{P}}$ and the wave conductivity of the magnetosphere $\Sigma_{\mathrm{A}}$ (Lyatsky and Maltsev, 1983). Theoretical estimates of electrodynamical magnetosphere-ionosphere interaction are usually made under the assumption that the ionospheric conductivity is high $\left(\Sigma_{\mathrm{P}} \gg\right.$ $\left.\Sigma_{\mathrm{A}}\right)$ during daytime hours and is low $\left(\Sigma_{\mathrm{P}} \ll \Sigma_{\mathrm{A}}\right)$ during nighttime. Several effects connected with the changing of ionosphere conditions near sunrise (sunset) have been predicted theoretically:

- the damping decrement $\gamma$ of the field line oscillations must pass through its extreme value near the terminator, where $\Sigma_{\mathrm{P}} \simeq \Sigma_{\mathrm{A}}$ (Newton et al., 1978; Allan and Knox, 1979);

- near the terminator the effect of the Hall part of the induction current associated with the inductive electric field of compressional mode on Alfvén wave becomes

Copy right (C) The Society of Geomagnetism and Earth, Planetary and Space Sciences (SGEPSS); The Seismological Society of Japan; The Volcanological Society of Japan; The Geodetic Society of Japan; The Japanese Society for Planetary Sciences. significant (Yoshikawa and Itonaga, 1996);

- the structure of the wave field along a field line must be different during day and night hours (Hameiri and Kivelson, 1991). This may lead to a less effective excitation of nighttime odd harmonics by an external source;

- a large difference between the conductivities of conjugate ionospheres may cause the occurrence of oscillations with a specific field-aligned structure: with the node of electric field in the highly conductive ionosphere and the anti-node in the weakly-conductive one (so called quarter-wave oscillations, Allan, 1983).

Some peculiarities of ULF waves may appear in connection with the sharp horizontal gradient of the ionospheric conductivity in the near terminator zone, e.g., the distortion of the horizontal polarization of the oscillations (Glassmeier, 1984; Saka et al., 1982), and the decrease of the reflection coefficient of the Alfvén waves $R_{\mathrm{A}}$ (Polyakov, 1988). The excitation of localized Alfvén oscillations by the moving terminator might also be possible.

In the widely used approximation of the thin ionosphere and the uniform magnetosphere (Alperovich and Fedorov, 1992) the conductivities $\Sigma_{\mathrm{P}}$ and $\Sigma_{\mathrm{A}}$ are formally independent. However, in reality the field-aligned plasma distribution in the magnetosphere is coupled with the ionospheric vertical profile. Besides, no analytical expression can be obtained for $Q$ in realistic non-uniform magnetospheric conditions. Other models of the magnetospheric resonator with a realistic distribution of the ionospheric and magnetospheric 
plasmas (e.g. Poulter et al., 1988) do not tackle the resonator's dissipative properties. In the present paper, with the help of numerical calculations of the parameters of the Alfvén resonator based on semi-empirical models of the plasma distributions in the magnetosphere-ionosphere system, the diurnal variations of the $Q$-factor are analyzed. The theoretical results are compared with those obtained from the analysis of the intensity of Pc 3-4 pulsations at mid- and low-latitude stations.

\section{Numerical Model of the Magnetosphere-Iono- sphere Resonator}

Our model of the magnetospheric Alfvén resonator which is based on the numerical solution of the equations for the eigen Alfvén oscillation of plasma shells. From the system of magnetohydrodynamic and Maxwell's equations, the coupled equations describing Alfvén and compressional modes can be derived. The spectral features of an Alfvén continuum are described by two sets of local ordinary differential equations along a field line (see short description of the results of spectral theory of MHD resonators in Pilipenko and Fedorov (1994)). The toroidal Alfvén mode corresponds to a disturbance which is more localized in a radial direction than in an azimuthal direction, or to a disturbance with a finite azimuthal wave number $m$, such that $m Q^{-1}\left(a / 2 L R_{\mathrm{E}}\right) \ll 1(a$ is the scale of plasma inhomogeneity in a radial direction). In a dipole geometry, the local dispersion equation for this mode is as follows (Yumoto et al., 1995)

$$
\begin{aligned}
\frac{\partial b_{\varphi}}{\partial x} & =i k_{0}\left(1+3 x^{2}\right) \epsilon_{\perp} e_{\nu}, \\
\frac{\partial e_{v}}{\partial x} & =i k_{0}\left(L R_{\mathrm{E}}\right)^{2} b_{\varphi} .
\end{aligned}
$$

Here $k_{0}=\omega / c$ is the vacuum wave number, $\omega=\omega_{\mathrm{A}}-i \gamma$ is the complex wave frequency. The azimuthal magnetic and radial electric covariant components of the wave field, $b_{\varphi}$ and $e_{\nu}$, are related to the physical components, $B_{\varphi}$ and $E_{\nu}$, by formulas which can be found in Yumoto et al. (1995). For a given latitude $\Phi$, the magnetic shell parameter $L$ is related to the inclination of the geomagnetic field $I$ by the relationship $\sin I=2[(L-1) /(4 L-3)]^{1 / 2}$. The field-aligned distributions of plasma and wave fields are presented in the form of dependencies on the variable $x=\sin \Phi$, whereas the coordinates of conjugate ionospheres are $x_{i}= \pm(1-1 / L)^{1 / 2}$. The complex dielectric permittivity $\epsilon_{\perp}$ incorporates both the dielectric permittivity and the local Pedersen conductivity $\sigma_{\mathrm{P}}$ of the magnetospheric-ionospheric plasma, i.e.

$$
\epsilon_{\perp}=\left(\frac{c}{C_{\mathrm{A}}}\right)^{2}+i \frac{4 \pi \sigma_{\mathrm{P}}}{\omega}
$$

where $C_{\mathrm{A}}$ is the Alfvén velocity. Within the approximation of a thin ionosphere the boundary condition for Alfvén oscillations is given by the well-known impedance relationship (Newton et al., 1978)

$$
B_{\varphi}=\frac{4 \pi \Sigma_{\mathrm{P}}}{c \sin I} E_{\nu} .
$$

If the field-aligned plasma distribution is assumed to be $N(r)=N_{0} \cos ^{-2 q} \Phi$, where $N_{0}$ is the plasma density at the magnetospheric equator, then the system (1) is reduced to

$$
\frac{\partial^{2} e_{v}}{\partial x^{2}}+\left(\frac{\omega}{\Omega_{\mathrm{A}}}\right)^{2}\left(1-x^{2}\right)^{6-q} e_{\nu}=0
$$

where $\Omega_{\mathrm{A}}=C_{\mathrm{A}}^{(0)} / L R_{\mathrm{E}}$, and $C_{\mathrm{A}}^{(0)}$ is the Alfvén velocity at the magnetospheric equator. In the "homogeneous" magnetosphere case, i.e. $q=6$, Eq. (4) becomes an equation with constant coefficients, though the $C_{\mathrm{A}}(x)$ distribution is still weakly inhomogeneous. Then, one can easily calculate analytically the main parameters of the magnetospheric resonator: eigenfrequency $\omega_{\mathrm{A}}$ of the $n$-th harmonic, $Q$-factor, reflection coefficient $R_{\mathrm{A}}$, and wave conductivity of the magnetosphere $\Sigma_{\mathrm{A}}^{(0)}$ (Yumoto et al., 1995). For the symmetric ionospheres we have

$$
\begin{gathered}
\omega_{\mathrm{A}}=\left(\frac{\pi}{2 x_{i}}\right) n \Omega_{\mathrm{A}}, \quad n=0, \pm 1, \ldots, \\
Q=\left(\frac{2 \gamma}{\left|\omega_{\mathrm{A}}\right|}\right)^{-1}=-\left(\frac{\pi|n|}{2}\right) \ln ^{-1}\left|R_{\mathrm{A}}\right|, \\
R_{\mathrm{A}}=\frac{\Sigma_{\mathrm{P}}-\Sigma_{\mathrm{A}}^{(0)} \sin I}{\Sigma_{\mathrm{P}}+\Sigma_{\mathrm{A}}^{(0)} \sin I}, \\
\Sigma_{\mathrm{A}}^{(0)}=\frac{c^{2}}{4 \pi C_{\mathrm{A}}^{(0)}\left(1+3 x_{i}^{2}\right)^{1 / 2}} .
\end{gathered}
$$

In the "homogeneous" magnetosphere case the damping rate of Alfvén oscillation is completely controlled by the ratio between the ionospheric conductivity and the wave conductivity of the "homogeneous" magnetosphere. In this particular case, $\Sigma_{\mathrm{A}}^{(0)}$ is determined by the value of the magnetospheric Alfvén velocity just above the ionosphere. For the marginal condition $\Sigma_{\mathrm{P}}=\Sigma_{\mathrm{A}}^{(0)} \sin I$, the Alfvén waves are totally absorbed by the ionosphere, i.e. $\gamma \rightarrow \infty$ and $Q \rightarrow 0$. However, in a more general situation ("inhomogeneous" magnetosphere, i.e. $q<6$ ) damping rates are modified due to a partial reflection of the Alfvén wave from field-aligned plasma inhomogeneities. Then, in contrast with Eq. (5), the damping rate has a non-vanishing value, i.e. $Q \neq 0$, for all the range of $\Sigma_{\mathrm{P}}$ values.

The field-aligned wave field structure has a different form for three possible sets of the conjugate ionospheres parameters:

- if in both hemispheres $\Sigma_{\mathrm{P}} \gg \Sigma_{\mathrm{A}}^{(0)} \sin I$, the wave field structure corresponds to the "fixed-end" case with nodes of electric field at the ionospheric boundaries;

. in the opposite case, when $\Sigma_{\mathrm{P}} \ll \Sigma_{\mathrm{A}}^{(0)} \sin I$ in both hemispheres, nighttime oscillations with "free-end" structure with anti-nodes of the electric field are to be observed;

. in the asymmetric case, when $\Sigma_{\mathrm{P}}^{\text {winter }} \ll \Sigma_{\mathrm{A}}^{(0)} \sin I \ll$ $\Sigma_{\mathrm{P}}^{\text {summer }}$, "quarter-wave" oscillations become possible. Instead of Eq. (5), the frequency of these oscillations is determined by the following:

$$
\omega_{\mathrm{A}}=\left(\frac{\pi}{2 x_{i}}\right) \frac{2 n-1}{2} \Omega_{\mathrm{A}} .
$$



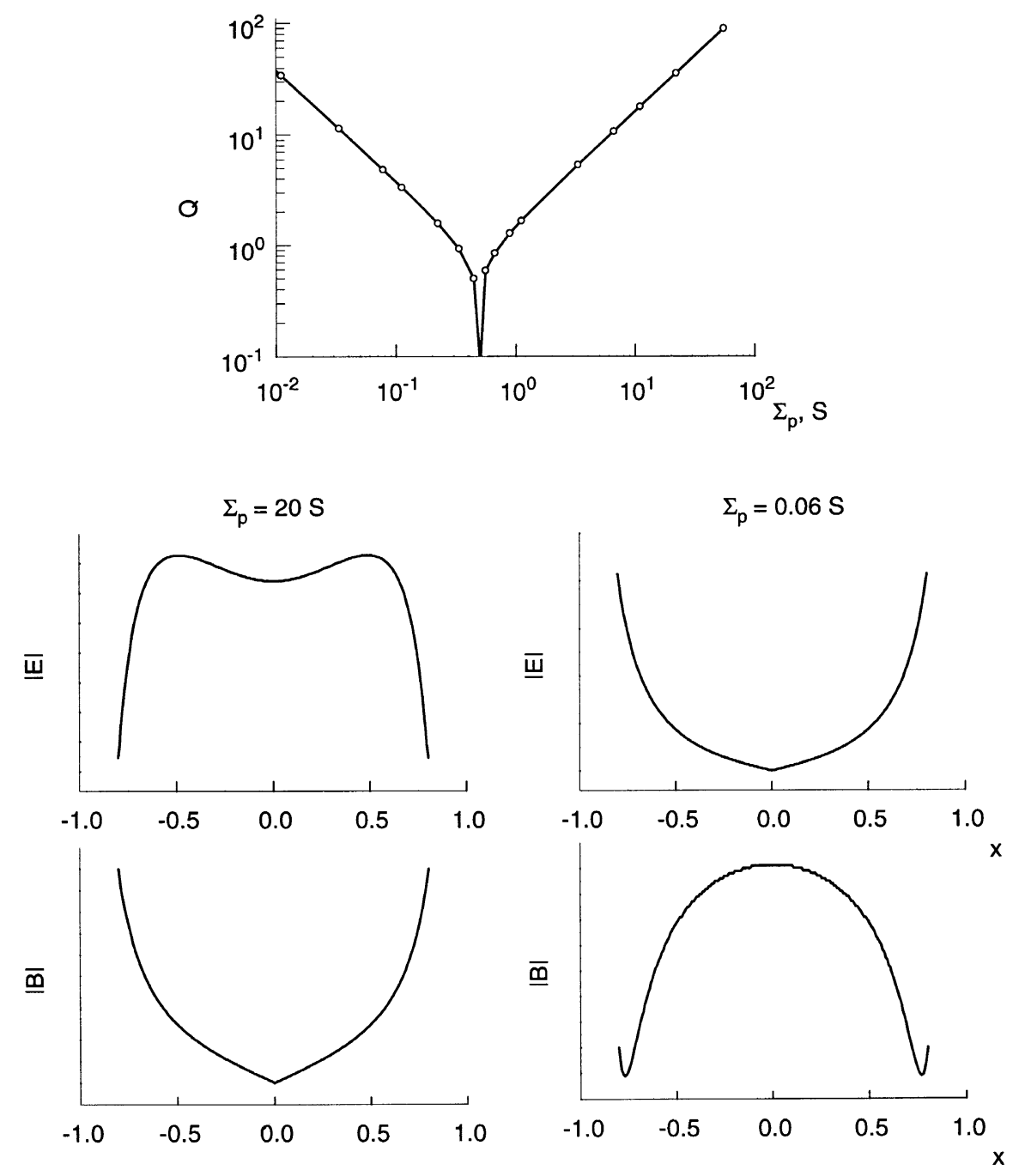

Fig. 1. Dependence of the $Q$-factor of the magnetospheric Alfvén resonator on the height-integrated Pedersen conductivity at $L=2.85$ for the "homogeneous" magnetosphere $(q=6)$ and "thin" ionosphere. Other model parameters are: $N_{0}=1.1 \cdot 10^{3} \mathrm{~cm}^{-3}, C_{\mathrm{A}}^{(0)}=9 \cdot 10^{2} \mathrm{~km} / \mathrm{s}$, and $f=15.4 \mathrm{mHz}$. Lower panels show field-aligned structure of magnetic and electric components (in arbitrary units) for high (left) and low (right) conductivities.

The lowest "quarter-wave" mode should have frequency nearly 2 times less than a first harmonic between symmetric ionospheres.

Under "fixed-end" boundary conditions the first harmonic (fundamental mode) corresponds to $n=1$ in Eq. (5). Under the "free-end" conditions this mode transforms into the aperiodically damping zero-mode $(n=0)$ (Newton et al., 1978). Hence, at nightside the lowest frequency mode, which may be experimentally observed, is the first harmonic, corresponding to $n=1$.

To illustrate the possible features of the magnetospheric Alfvén resonator for the "thin" ionosphere and "homogeneous" magnetosphere $(q=6)$, we present in Fig. 1 the dependence of the $Q$-factors of the fundamental modes $(n=1$, $f=15 \mathrm{mHz}$ ) on the ionospheric conductivity $\Sigma_{\mathrm{P}}$. Calculations have been done for the following set of magnetospheric parameters: $L=2.85, N_{0}=1.1 \cdot 10^{3} \mathrm{~cm}^{-3}$, and $C_{\mathrm{A}}^{(0)}=9.1 \cdot 10^{2} \mathrm{~km} / \mathrm{s}$. The magnetospheric wave conductance for this set of parameters is $\Sigma_{\mathrm{A}}^{(0)}=0.5 \mathrm{~S}$ (remember that $\left.\Sigma(\mathrm{sm} / \mathrm{s})=9 \cdot 10^{11} \Sigma(\mathrm{S})\right)$. The field-aligned structure of first harmonic of standing Alfvén waves under the "fixed-end" boundary condition and the "free-end" condition is shown in the left and right bottom panels, respectively.

In an inhomogeneous magnetosphere the relationships (5) cannot be applied, and the notions of $R_{\mathrm{A}}$ and $\Sigma_{\mathrm{A}}$ have no obvious physical sense. However, we keep using them, but in a sense of some effective values, related to $Q$. The effective $\Sigma_{\mathrm{A}}$ means a critical value of the ionospheric conductance, which determines different types of a field-aligned wave structure. Under realistic geophysical conditions, the plasma density in the lower ionosphere, which determines the value of $\Sigma_{\mathrm{P}}$, and the plasma density above the ionosphere, which determines the $\Sigma_{\mathrm{A}}$, both vary in a coordinated way. As a result, diurnal variations of the $Q$-factor of the magnetospheric resonator can be different from those estimated within the framework of the "thin" ionosphere and the "homogeneous" magnetosphere model.

In the numerical model used here, a more realistic spatial distribution of magnetospheric-ionospheric plasma for a particular set of geophysical conditions is taken into account. 

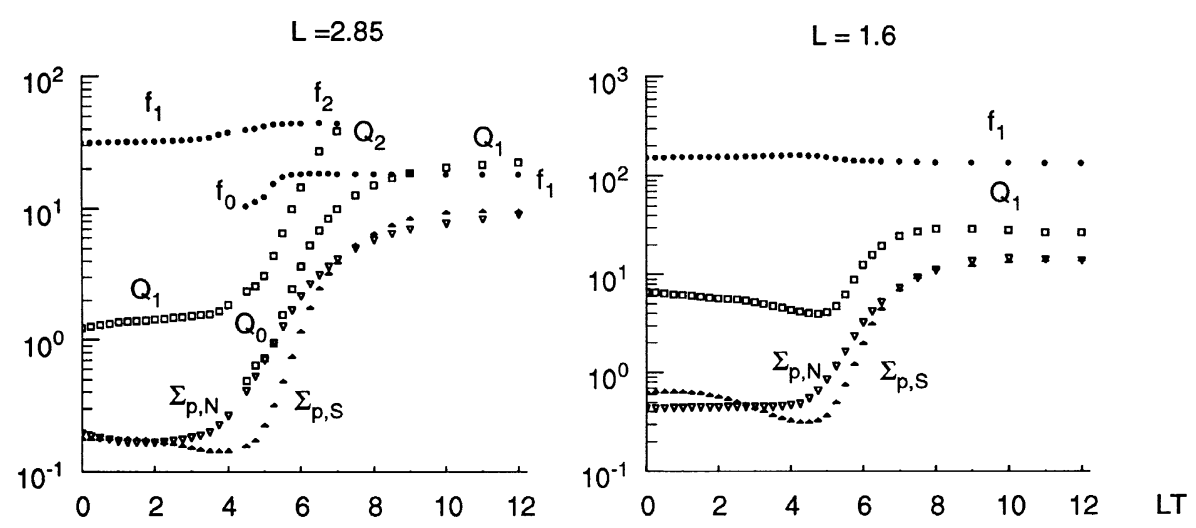

IEI
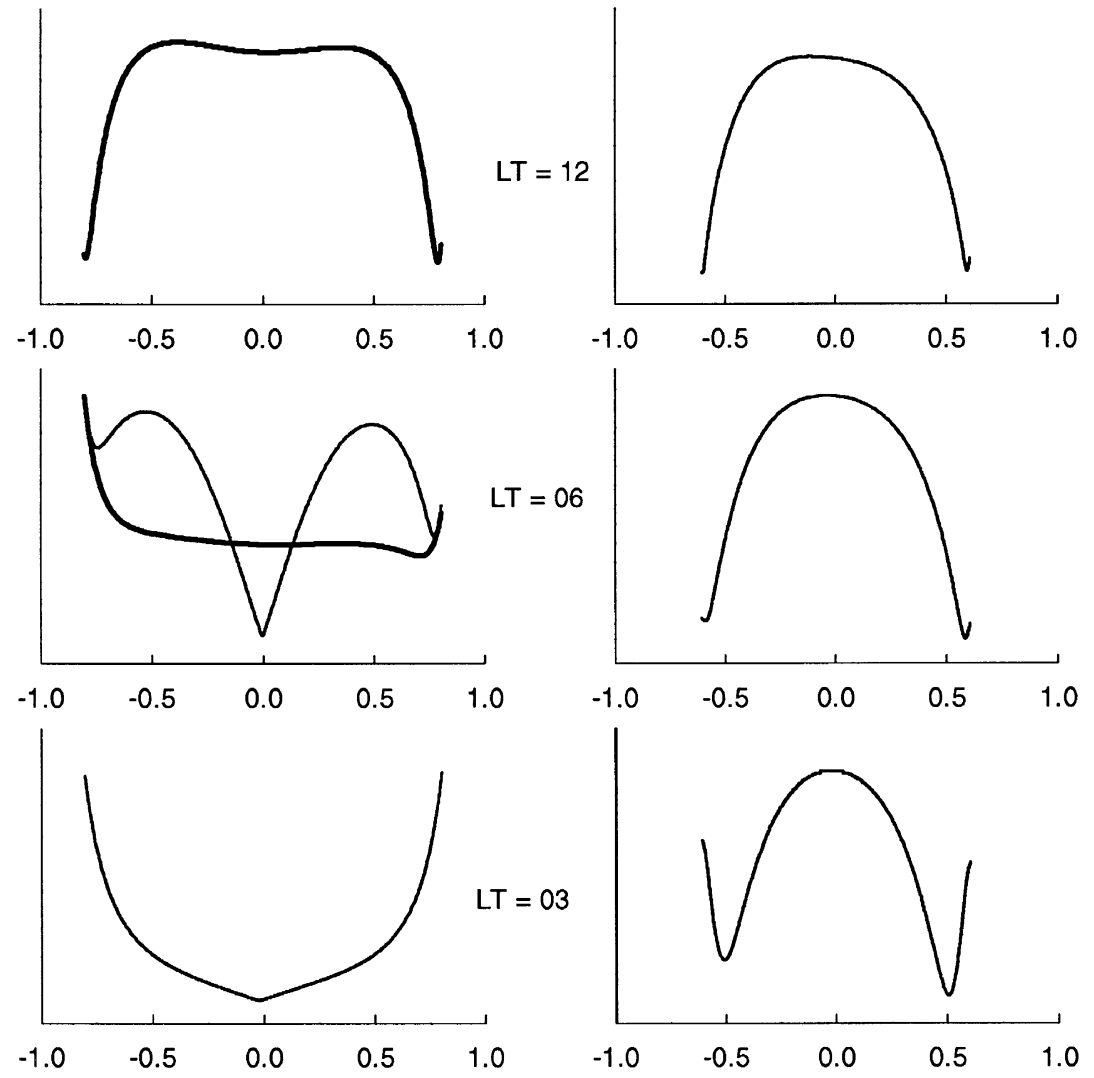

Fig. 2. Diurnal variations of the $Q$ factor (squares) and frequency $f(\mathrm{mHz})$ (dots) for the daytime and nighttime harmonics, and height-integrated Pedersen conductivities (triangles) of conjugate ionospheres at $L=2.85$ (left) and $L=1.6$ (right) (September, $R=10$ ). Lower panels show the field-aligned structure of electric component for the particular hour at daytime, sunrise and nighttime.

The empirical ionospheric model IRI-90 (Bilitza, 1992) has been used to obtain the plasma density below $650 \mathrm{~km}$. The electron density $N_{0}$ in the equatorial plane of the magnetosphere has been calculated from the empirical model of Carpenter and Anderson (1992), based on whistler and ISEE satellite data. The IRI vertical profile has been conjugated with the value of $N_{0}$ under the assumption of diffusive equilibrium along a field line (Angerami and Thomas, 1964). Following the model by Strangeways (1986), a power law for the electron/ion field-aligned temperature profiles has been adopted with some modifications in order to take into account the north-south ionospheric conductivity asymmetry. Using this ionosphere-magnetosphere plasma distribution model,
Eq. (1) have been solved with the boundary condition at the bottom of the ionosphere $j_{\|}\left(x= \pm x_{i}\right)=0$, which means the non-penetration of field-aligned current into an insulating atmosphere. The input parameters of the model are: geographic or geomagnetic coordinates, local time $L T$, day number and index of solar activity $R$.

The resonator parameters for the first three Alfvén harmonics and their field-aligned structures within the Pc 3-4 band have been calculated for $L$-shells from 1.6 to 4.0 . Massloading effects of the ionospheric ions at these latitudes can be neglected (Pilipenko et al., 1998). The geophysical situation corresponds to equinox and low solar activity $(R=10)$. Such low $R$ values are chosen to ensure the most favorable 
conditions for the dayside/nighttime change of wave structure and the occurrence of quarter-wave mode.

Figure 2 shows diurnal variations of the resonator spectral parameters: the frequency $f(\mathrm{mHz})$ and the $Q$-factor, and the height-integrated conductivities $\Sigma_{\mathrm{P}}^{(\mathrm{N})}$ and $\Sigma_{\mathrm{P}}^{(\mathrm{S})}$ for northern (N) and southern (S) conjugate ionospheres.

The calculation results for the middle latitude $(L=2.85)$ are presented in the left panel. The equatorial plasma density and Alfvén velocity are $N_{0}=8.7 \cdot 10^{2} \mathrm{~cm}^{-3}$, and $C_{\mathrm{A}}^{(0)}=$ $10^{3} \mathrm{~km} / \mathrm{s}$. In the Carpenter-Anderson model the value of $N_{0}$ inside the plasmasphere does not depend on $L T$ and $K_{p}$. From dayside to nightside hours the conductivity varies from $\Sigma_{\mathrm{P}}^{(\mathrm{N})} \simeq \Sigma_{\mathrm{P}}^{(\mathrm{S})} \sim 9 \mathrm{~S}$ to $\sim 0.2 \mathrm{~S}$, while the maximal values of $C_{\mathrm{A}}$ in the topside ionosphere changes from $\sim 6.5 \cdot 10^{3} \mathrm{~km} / \mathrm{s}$ to $\sim 9 \cdot 10^{3} \mathrm{~km} / \mathrm{s}$.

The "fixed-end" first harmonic (fundamental mode), having $Q_{1} \simeq 20$ at dayside, gradually transforms into the nonoscillatory zero-harmonic at nightside (Newton et al., 1978). The $Q$-factor of the daytime second harmonic, which gradually transforms into the nighttime first harmonic with the decrease of the ionospheric conductivity, drops from $Q_{2} \simeq 30$ at dayside to $Q_{1} \simeq 1.5$ in nighttime.

In the bottom panels of Fig. 2 the field-aligned structures of the wave electric field components $E_{v}(x)$ of the lowestfrequency modes for selected $L T$ are presented. Oscillation at $L T=12$ has a structure of the first "fixed-end" harmonic. At $L T=3$ (pre-dawn hours) the wave structure corresponds to a "free-end" first harmonic, which evidences the transition to values of ionospheric conductivities below critical ones, i.e., $\Sigma_{\mathrm{P}} \leq \Sigma_{\mathrm{A}}$. However, in the nighttime sector the $Q$ factor of this mode becomes low, that its resonant excitation is unlikely to be possible.

Similar calculations for the low latitude $(L=1.6)$ for the parameters $N_{0}=1.8 \cdot 10^{3} \mathrm{~cm}^{-3}$, and $C_{\mathrm{A}}^{(0)}=4 \cdot 10^{3} \mathrm{~km} / \mathrm{s}$ indicate a different behavior (right panel of Fig. 2): though the $Q$-factor of the "fixed-end" first harmonic drops several times during the transition from dayside to nightside conditions, but it still remains rather high, $Q_{1} \simeq 5$. The wave field-aligned structure in the magnetosphere does not change qualitatively during the entire day, which indicates that the relation $\Sigma_{\mathrm{P}}>\Sigma_{\mathrm{A}}$ holds both during the day and night hours. At $L T=3$ an enhancement of the ionospheric dissipative properties results in the upward shift of the apparent node of the first harmonic wave structure (Fig. 2, right-hand bottom panel).

The particular conditions, when $\Sigma_{\mathrm{P}}$ becomes comparable to $\Sigma_{\mathrm{A}}$, depends on local time, latitude, solar activity, and harmonic number. The calculations show that for a wide range of geophysical parameters the ionosphere at low latitudes ( $L \leq 2)$ keeps a high conductivity $\left(\Sigma_{\mathrm{P}}>\Sigma_{\mathrm{A}}\right)$ for Alfvén oscillations during the entire day. The numerical results show that a critical value of $\Sigma_{\mathrm{A}}$, when $Q \leq 1$, is $\Sigma_{\mathrm{A}} \simeq 0.2-0.4 \mathrm{~S}$, i.e., lower than a value usually used for the analytical estimates. The consequence of this fact is that for typical $\Sigma_{\mathrm{P}}$ the first "free-end" harmonic at nightside never occurs in a low-damping regime, when $\Sigma_{\mathrm{P}} \ll \Sigma_{\mathrm{A}}$.

In the "homogeneous" magnetosphere case, described by the relationships (5), the damping rate $\gamma$ does not depend on harmonic number $n$. In a realistic situation this dependence can be different. Figure 3 shows the calculated diur-
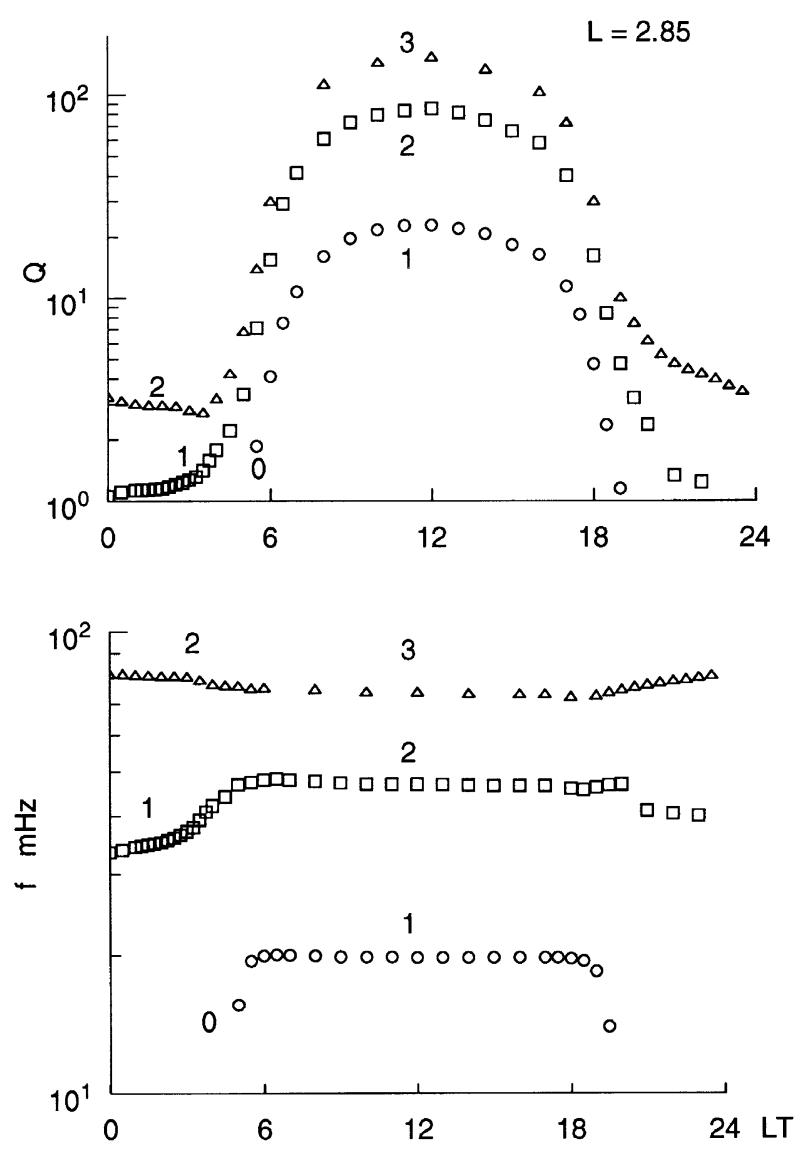

Fig. 3. Diurnal variations of the $Q$-factors (upper plot) and frequencies (lower plot) at $L=2.85$ for the first three harmonics of the magnetospheric resonator.

nal variations of $Q$-factors (a) and eigenfrequencies (b) for the first three harmonics at $L=2.85$. The 1 -st harmonic ( $f_{1} \simeq 20 \mathrm{mHz}$ ) of dayside oscillations transforms in the aperiodic mode during the transition to nightside conditions. The dayside second harmonic $\left(f_{2} \simeq 30 \mathrm{mHz}\right.$ ), which transforms into the first harmonic at the nightside, turns out to be highly damped, i.e., $Q_{1} \simeq 1$. Meanwhile the third dayside harmonic ( $f_{3} \simeq 80 \mathrm{mHz}$ ), transforming into nightside second harmonic, keeps a rather high $Q$-factor, $Q_{2} \geq 3$, during the entire night. Hence, the higher $n$ harmonics should be excited by the same source more easily.

\section{Influence of the Ionospheric Hall Conductivity on Magnetospheric Resonant Oscillations}

Our numerical model does not use as an input parameter the vertical profile of the Hall conductivity of the ionosphere. Recently, Yoshikawa and Itonaga (1996) claimed the necessity of taking into account the inductive Hall effects for the calculation of parameters of magnetospheric field line oscillations for large Hall conductivities of the ionosphere, i.e. $\Sigma_{\mathrm{H}}>\Sigma_{\mathrm{P}}$. In general, this problem tackles rather complicated theoretical aspects of spectral features of MHD disturbances in inhomogeneous and gyrotropic plasma systems. Here we just briefly discuss the possible range of magnetospheric and ionospheric parameters where the Hall conductivity has no noticeable influence on the coefficient of an 

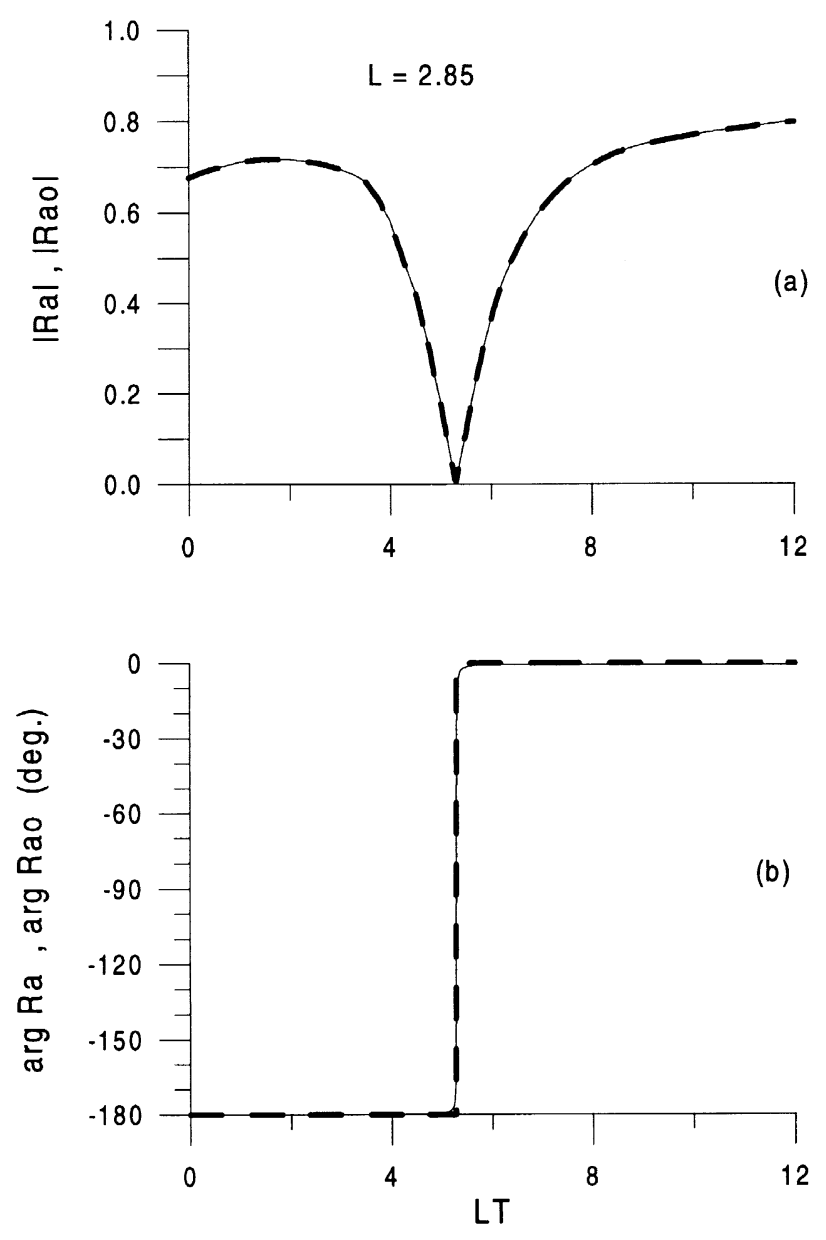

Fig. 4. Diurnal variations of the absolute values (a) and phases (b) of the reflection coefficients, $R_{\mathrm{A}}$ and $R_{\mathrm{A}}^{(0)}$, of an Alfvén wave from the thin ionospheric layer with account for Hall conductivity (solid thin line) and neglecting it (dashed thick line).

Alfvén wave reflection from the ionosphere.

We have estimated numerically the relative role of inductive Hall currents on the reflection features of an Alfvén wave at middle latitudes. Figure 4 shows the diurnal variations of the absolute values (a) and phases (b) of the coefficients of an Alfvén wave reflection from the "thin" ionospheric layer for the horizontal wave number $k=10^{-2} \mathrm{~km}^{-1}$ with the account for the Hall conductivity, $R_{\mathrm{A}}$ (solid thin line), and neglecting it (5), $R_{\mathrm{A}}^{(0)}$ (dashed thick line). Both height-integrated conductivities, $\Sigma_{\mathrm{P}}$ and $\Sigma_{\mathrm{H}}$, are calculated with the use of IRI model for the same geophysical conditions as in Fig. 2. Throughout the entire day the deviation between absolute values of $\left|R_{\mathrm{A}}\right|$ and $\left|R_{\mathrm{A}}^{0}\right|$ does not exceed $0.1 \%$, and only within narrow time intervals near the sunrise and the sunset this deviation slightly increases to $\leq 1 \%$. The difference between phases, $\delta \phi=\left|\arg R_{\mathrm{A}}-\arg R_{\mathrm{A}}^{(0)}\right|$, practically everywhere is less than $2^{\circ}$, besides the narrow interval from 5:20 to 5:40 LT near the terminator where $\delta \phi$ can be $\sim 20^{\circ}$.

These calculations prove that at middle latitudes the influence of ionospheric Hall conductivity on the reflection coefficient, and, therefore, on frequency and damping rate, of resonant Alfvén oscillations can be neglected. The analysis made in Appendix shows that the approximate formula (5) for the reflection coefficient can be used for Hall conductivity $\Sigma_{\mathrm{H}} \leq \Sigma_{\mathrm{H}}^{*}$, where

$$
\Sigma_{\mathrm{H}}^{*} \simeq\left(\frac{k C_{\mathrm{A}}}{\omega}\left|\Sigma_{\mathrm{P}}^{2}-\Sigma_{\mathrm{A}}^{2}\right|\right)^{1 / 2} .
$$

For a typical resonant oscillations in the Pc 3-4 range the estimate Eq. (7) gives $\Sigma_{\mathrm{H}}^{*} \simeq 10 \Sigma_{\mathrm{P}}$.

\section{Diurnal Variations of Pc 3 Parameters}

For experimental verification of some conclusions stemming from the model calculations we have used data from stations along the $210^{\circ}$ magnetic meridian: Magadan (MGD, $L=2.85$ ), Paratunka (PTK, $L=2.11$ ), Moshiri (MSR, $L=1.61)$ and Onagawa (ONW, $L=1.38)$ in the northern hemisphere and Adelaide (ADL, $L=2.13$ ), Birdsville (BSV, $L=1.57$ ) and Weipa (WEP, $L=1.18$ ) in the southern hemisphere. More detailed characteristics of the fluxgate magnetometers installed at the " $210^{\circ}$ Magnetic Meridian" network are given by Yumoto et al. (1992, 1996). Additionally, we have used data from the induction magnetometer at station l'Aquila (LAQ), situated at the same geomagnetic latitude as MSR, $L=1.6$ (Vellante et al., 1989).

We analyzed the averaged spectral intensities of the $H$ component of Pc 3-4 waves in the morning hours. The analyzed interval October 13-17, 1992 is characterized by a rather high ULF wave activity. Spectral estimates have been performed using the Blackmann-Tukey method in a moving 15-min window. The background spectral power in the range 10-100 $\mathrm{mHz}$ has a colored-noise form $H(f) \sim f^{-\alpha}$. During the 5 days considered the average value of the parameter $\alpha$ was 1.5 for all stations at $1.6<L<3$. In a "pre-whitened" spectrum $H_{\mathrm{W}}(f)=H(f) \cdot f^{\alpha}$ the background slope is excluded. Hence, the averaged variations of $H_{\mathrm{W}}$ in a given frequency band $\left\langle H_{\mathrm{W}}\right\rangle_{f}(L T)$ for a particular day number characterize more adequately the variations of ULF activity. To reveal systematic effects in the diurnal variations of spectral intensity, the values $\left\langle H_{\mathrm{W}}\right\rangle_{f}(L T)$ have been averaged over 5-day period. For the time period considered the variations of sunrise moments are small and have not been accounted for.

The model predicts different dependencies of the dissipation rates of different Alfvén harmonics on the ionospheric conductivity (Fig. 3). The relevant analysis of the experimental data at MGD $(L=2.85)$, and at BSV/LAQ $(L \approx 1.6)$ are shown in Fig. 5. Figure 5(a) demonstrates the aftersunrise enhancement of ULF activity at the mid-latitude station MGD $(L=2.85)$. The function $\left\langle H_{\mathrm{W}}\right\rangle_{f}(L T)$ has been calculated in the two frequency bands: $10-30 \mathrm{mHz}(\mathrm{LF})$ and 60-100 $\mathrm{mHz}(\mathrm{HF})$. The after-sunrise enhancement of the ULF intensity can be seen in both frequency bands, but at lower frequency (LF) it is stronger. According to the numerical calculations with a realistic ionosphere model, the low frequency band (LF) should correspond to the first harmonic of daytime Alfvén oscillations, whereas the high frequency one (HF) is related to the third harmonic. The experimental data agree with the results of model calculations (Fig. 3), predicting a weaker dependence of higher harmonics on the ionospheric conductivity.

Another experimental evidence which confirms this conclusion can be found in Waters et al. (1991). Three harmonics 


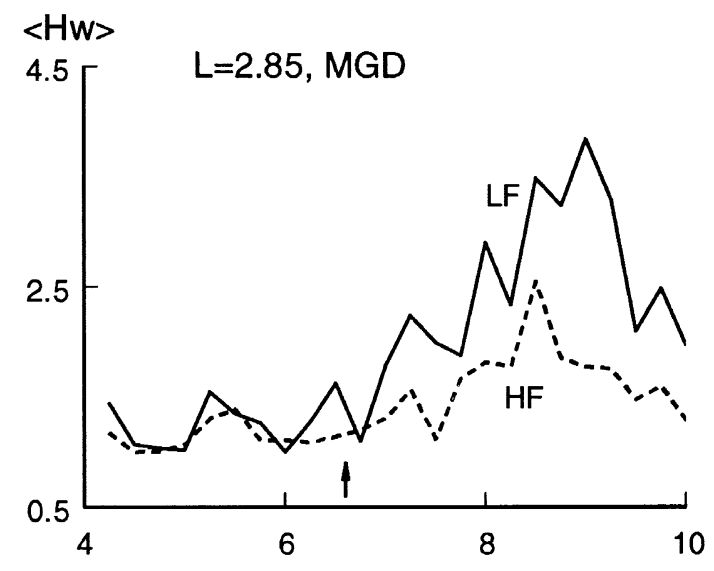

(a)

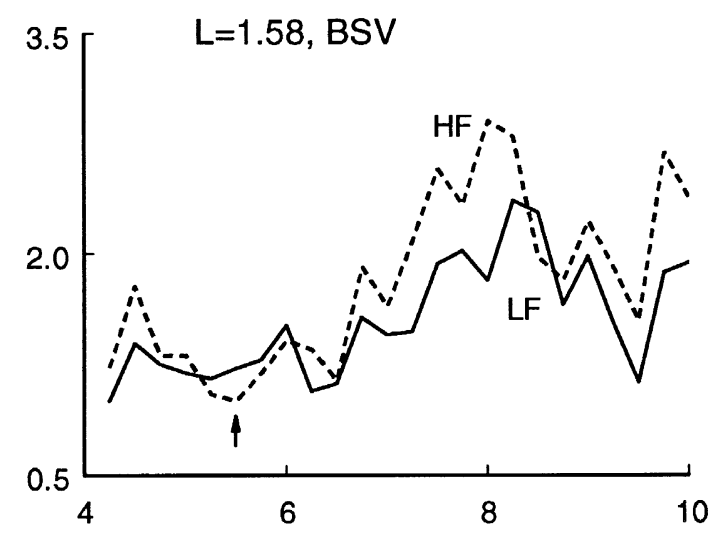

(b)

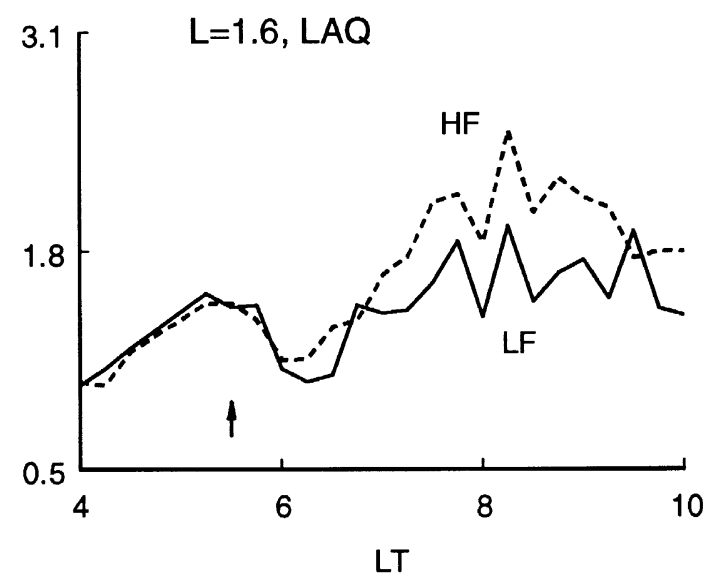

(c)

Fig. 5. Intensities $\left\langle H_{\mathrm{W}}\right\rangle$ (in arbitrary units) of Pc 3 pulsations measured in the HF and LF bands, averaged over 5 successive days (October 13-17, 1992). Each point corresponds to a $15-\mathrm{min}$ interval. Stations and frequency bands are as follows: (a) MGD, $L=2.85,10-30 \mathrm{mHz}$ (LF) and 60-100 mHz (HF); (b) BSV, $L=1.57$ and (c) LAQ, $L=1.6$; for both latter stations $30-60 \mathrm{mHz}(\mathrm{LF})$ and $70-110 \mathrm{mHz}(\mathrm{HF})$. Arrows indicate moments of sunrise in the local ionospheres. were detected with the help of gradient cross-phase analysis at the pair of stations located at $L \simeq 2$.6. The third harmonic ( $f_{3} \simeq 60 \mathrm{mHz}$ ) became detectable at $\sim 4$ LT, i.e. nearly two hours earlier than the two lower harmonics.

The spectral content of ULF pulsations is determined both by an external source and a local resonant response, the latter being dependent on the $Q$-factor of the magnetospheric resonator. The transmission of an Alfvén wave through the ionosphere to the ground is determined primarily by the factor $\Sigma_{\mathrm{H}} / \Sigma_{\mathrm{P}}$ (Hughes and Southwood, 1976), which is rather stable during the entire day and remains practically the same in both the daytime and nighttime ionospheres. According to this consideration, the influence of the ionospheric conductivity variations on ULF waves intensity should be different in frequency bands near a local resonant frequency and beyond it. We apply the same procedure of data analysis to the morning Pc 3 pulsations at the low-latitude stations at $L=1.6: \operatorname{BSV}\left(\lambda=139^{\circ}\right)$ and $\operatorname{LAQ}\left(\lambda=17^{\circ}\right)$, differing by nearly 8 hours in local time. The comparison of two frequency ranges: $30-60 \mathrm{mHz}(\mathrm{LF})$ and $70-110 \mathrm{mHz}$ (HF) demonstrates the stronger after-dawn enhancement in the high frequency band (Figs. 5(b) and (c)). According to model calculations and special experimental investigations (Baransky et al., 1995; Vellante et al., 1996; Pilipenko et al., 1997) the frequency of the fundamental mode of the Alfvén oscillations at this geomagnetic shell falls within the interval $60-100 \mathrm{mHz}$. The regularly observed oscillations with lower frequencies in the range $30-60 \mathrm{mHz}(\mathrm{LF})$ are not related to a local field line resonance. So, we conclude that the difference in pulsations variations in two frequency bands, (LF) and (HF), after the moment of sunrise is caused by the influence of ionospheric conductivity on the $Q$-factor of Alfvén oscillations and really reveals itself mainly at a frequency of the field line resonant oscillations.

Another conclusion following from the calculations with a realistic ionosphere model is that the influence of the ionospheric dissipation on the magnetospheric Alfvén resonator at lower latitudes is weaker (Fig. 2). In fact, the comparison of the pulsation intensities at resonant frequency bands at stations with different $L$ shows that the after-sunrise increase is higher at the mid-latitude station than at the low-latitude one. As an example, the diurnal variations of the averaged normalized ULF intensity at the stations MGD and BSV in the frequency bands corresponding to local fundamental harmonics, 10-30 mHz (LF) and 60-100 mHz (HF), are shown in Fig. 6. The ratio of the after-sunrise pulsation intensity to the pre-sunrise one is $\sim 2$ at MGD and $\sim 1.6$ at BSV. Therefore, the theoretical predictions about a weaker influence of the ionospheric dissipation on the magnetospheric Alfvén resonator at lower latitudes is in a qualitative agreement with the experimental results.

Strictly speaking, the fading of ULF intensity at pre-dawn hours could be caused not only by the variations of ionospheric conductivity, but by the attenuation of driving compressional disturbances during their tailward propagation also. In order to demonstrate unambiguously once again the role of local resonant properties of the magnetosphericionospheric system, we consider the Pc 3 event detected from 18 to $24 \mathrm{UT}(\mathrm{LT}=\mathrm{UT}+9)$ on October 17, 1992. The feature of this event is the stability of the central fre- 


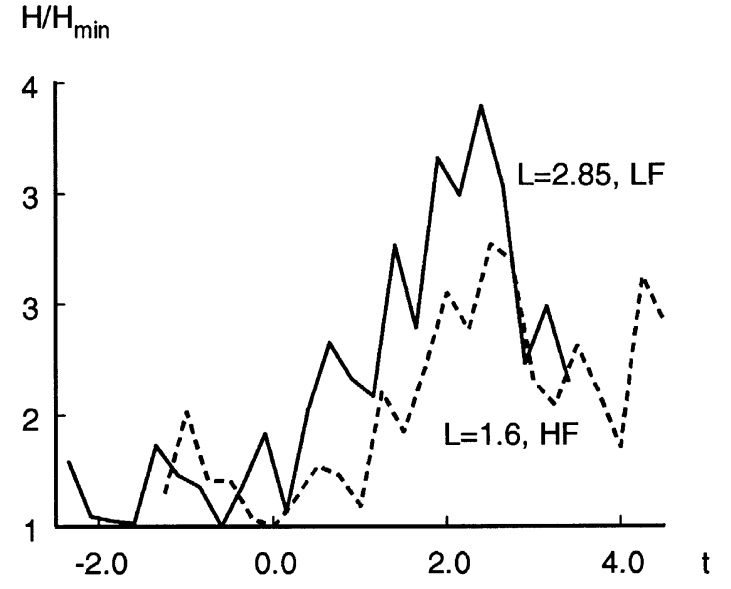

Fig. 6. The time dependence of the relative intensity of Pc 3 pulsations recorded at $\operatorname{MGD}(L=2.85)$ and $\operatorname{BSV}(L=1.57)$ in frequency bands corresponding to local Alfvén resonances, measured from the moment of ionospheric sunrise $(t=0)$.

quency, $f \simeq 40 \mathrm{mHz}$, of the pulsations observed throughout all the meridional profile of the $210^{\circ} \mathrm{MM}$ stations in both hemispheres (a similar event was discussed by Ziesolleck et al. (1993)). The central frequency is in good accordance with that calculated from the relationship $f=6.25 \cdot B$ (Gugliel'mi, 1988) for the simultaneous IMF magnitudes $B=5.6-6.1 \mathrm{nT}$ and, probably, it corresponds to the frequency of upstream waves. Figure 7 demonstrates the temporal variations of the spectral amplitude $\langle H(f)\rangle$ at the frequency band $30-50 \mathrm{mHz}$, averaged over a $15-\mathrm{min}$ interval. Variations of $\langle H(f)\rangle$ at 4 stations in the northern hemisphere are shown in the upper panel, whereas variations at 3 stations in the southern hemisphere are shown in the bottom plot. For all the stations, the pulsation amplitude increases after sunrise (20-21 UT) and decreases at pre-noon hours. But, at $L \simeq 2.1$ (stations PTK and ADL), this effect is much stronger than at all the other stations. Although the central frequency of a probable source is almost the same throughout the event, the amplitude profile has a maximum at $L \simeq 2.1$, i.e. just at those stations where local resonant frequencies are close to the source frequency. In fact, the model calculation confirms that the local resonant frequency is $\sim 40 \mathrm{mHz}$ at $L \simeq 2.1$.

In our data set we have not observed any noticeable changes in the spectral content of Pc 3-4 pulsations during the crossing of the terminator. Dynamical spectral analysis has not shown any rapid variations of the ULF spectra which might be expected due to a possible excitation of the asymmetric quarter-wave mode, described by Eq. (6). According to the results of the numerical model this fact is not unexpected. Within the framework of our model the condition $\Sigma_{\mathrm{P}}^{(\mathrm{N})} \ll \Sigma_{\mathrm{A}} \ll \Sigma_{\mathrm{P}}^{(\mathrm{S})}$, which is necessary for the excitation of a quarter-wave mode, cannot hold at middle latitudes.

Also, we have not noticed any localized anomaly of Pc 3-4 amplitude which might be associated to the sunrise moment. From the results of the Itonaga and Kitamura (1988) model it follows that a disturbance due to an ionospheric inhomogeneity decays in the azimuthal direction on a scale comparable to the meridional wave length, $\lambda_{x}$, of the incident Alfvén wave.
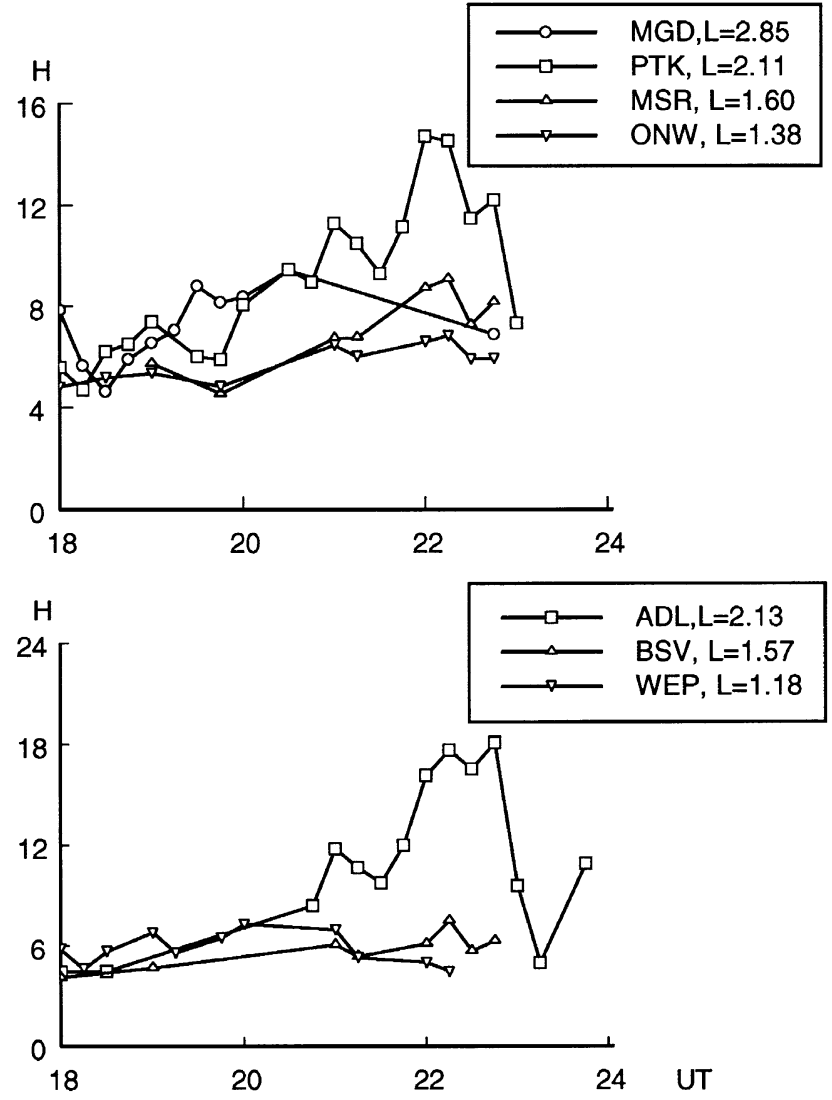

Fig. 7. Variations of spectral power of the $H$ component at the frequency band $30-50 \mathrm{mHz}$ at the $210^{\circ} \mathrm{MM}$ stations in the northern (upper) and southern (lower) hemispheres during morning hours on October 17, 1992.

Therefore, when $\lambda_{x}$ is sufficiently smaller that the azimuthal scale of an ionospheric inhomogeneity, $\Delta l$, the ionosphere can be considered as uniform for these ULF waves. The IRI model predicts that at middle latitudes $\Delta l \simeq 1^{h} \simeq 10^{3} \mathrm{~km}$. According to numerous experimental studies of the Pc 3-4 resonant structure (e.g. Baransky et al., 1995) $\lambda_{x} \simeq 300 \mathrm{~km}$, hence the inequality $\lambda_{x}<\Delta l$ holds. This consideration may explain the absence of the sunrise effects in our data: the gradient of the ionospheric conductivity is not steep enough to cause a significant distortion of the ground ULF wave structure.

\section{Conclusion}

The analysis of a realistic numerical model of the magnetospheric-ionospheric Alfvén resonator demonstrates different forms of diurnal variations of Pc 3-4 ULF waves at low and middle latitudes. We have proved that there is no necessity to take into account the inductive Hall effects for the calculation of the Alfvén resonator parameters at typical mid-latitude conditions. At all latitudes within $L=1.5-$ 3.0, the transition to the regime of the low-damping "freeend" oscillations $\left(\Sigma_{\mathrm{P}} \ll \Sigma_{\mathrm{A}}\right)$ is not realized. Thus, it seems that in a realistic magnetosphere any effects related to the change of the field-aligned structure of eigen oscillations during the transfer from night to day hours are not likely to be observed. Meanwhile, at low latitudes $(L<2)$, during the whole day the ionosphere remains "highly-conductive" 
for an incident Alfvén wave, i.e. the condition $\Sigma_{\mathrm{P}}>\Sigma_{\mathrm{A}}$ holds. At higher latitudes $(L>2)$ at night hours a fundamental Alfvén mode occurs in a regime of strong damping $\left(\Sigma_{\mathrm{P}} \leq \Sigma_{\mathrm{A}}\right)$ and, consequently, the ULF signals at this frequency should correspond to forced oscillations. The resonant response at these latitudes can be expected only at higher harmonics $(n \geq 2)$. The difference between the behavior of $Q$-factors in the simplified thin ionosphere model and in the more realistic model discussed above is caused by the fact that the effective wave conductance of the magnetosphere, determined by a field-aligned plasma distribution, in fact is lower than it was expected from simplified estimates. A coordinated variation of densities of the ionospheric and magnetospheric plasmas makes a resonant excitation of the asymmetric "quarter-wave" mode within the plasmasphere impossible.

The predictions of the numerical model on the $L T$ dependence of the $Q$-factors of the magnetospheric resonator are in a qualitative agreement with the character of the diurnal variations of Pc 3-4 ULF waves. In frequency bands corresponding to the field line Alfvén resonances, especially their fundamental harmonics, the influence of the ionosphere variations is more pronounced than in non-resonant bands. With the help of the calculation results, it is possible to explain the difference in diurnal variations of ULF intensity in various frequency sub-bands and at different latitudes.

Acknowledgments. This research was supported by INTAS (grant 93-3412). Our sincere thanks go to all the member of the $210^{\circ} \mathrm{MM}$ Magnetic Observation Project for their ceaseless support. The editor thanks S. Fujita and B. J. Fraser for their assistance in evaluating this paper.

\section{Appendix. Estimate of Critical Hall Conductivity}

Yoshikawa and Itonaga (1996) estimated a possible influence of the Hall conductivity on resonant Alfvén oscillations of the magnetosphere through the analysis of the coefficient of Alfvén waves reflection from the ionosphere. Following their approach, we find from a physically evident consideration a condition, under which the Hall conductivity has no effect on the reflection of Alfvén waves from the ionosphere.

Let an Alfvén wave with the amplitude $B_{y}^{(i)}$ impinges on a thin ionospheric sheet with the height-integrated conductivities $\Sigma_{\mathrm{P}}$ and $\Sigma_{\mathrm{H}}$, situated at the height $h$ above an infinitely conductive ground. The axes $x$ and $y$ are directed northward and westward, correspondingly. Magnetic field is vertical, i.e. $I=\pi / 2$. From formulae for the reflection coefficient (5) and the boundary condition (3) we find in the zero-th approximation the wave electric field above the ionosphere

$$
E_{x}=-\frac{c}{2 \pi} \frac{B_{y}^{(i)}}{\Sigma_{\mathrm{P}}+\Sigma_{\mathrm{A}}} .
$$

The height-integrated Hall current, $J_{\mathrm{H} y}=-\Sigma_{\mathrm{H}} E_{x}$, excited by this field, generates a fast compressional mode with the magnetic field component

$$
B_{x}=\frac{4 \pi}{c} \frac{J_{\mathrm{H} y}}{1+\operatorname{coth}|k| h}=\frac{2}{1+\operatorname{coth}|k| h} \frac{\Sigma_{\mathrm{H}}}{\Sigma_{\mathrm{P}}+\Sigma_{\mathrm{H}}} B_{y}^{(i)} .
$$

In its turn, the non-steady magnetic field (A.2) induces the inductive electric field $\widetilde{E}_{y}$. From the Faraday law and the relationship $\widetilde{E}_{y} \propto \exp (-|k| z)$, we obtain that $\widetilde{E}_{y}=i\left(k_{0} /|k|\right) B_{x}$.
The Hall current produced by the inductive electric field is given by $\widetilde{J}_{\mathrm{H} x}=\Sigma_{\mathrm{H}} \widetilde{E}_{y}$. Substituting in the latter relationship the expression for $\widetilde{E}_{y}$, we find that $\widetilde{J}_{\mathrm{H} x}=i\left(k_{0} /|k|\right) \Sigma_{\mathrm{H}} B_{x}$. Replacing $B_{x}$ with the use of Eq. (A.2), we obtain from the latter relationship that

$$
\tilde{J}_{\mathrm{H} x}=i \frac{2 k_{0}}{|k|(1+\operatorname{coth}|k| h)} \frac{\Sigma_{\mathrm{H}}^{2}}{\Sigma_{\mathrm{P}}+\Sigma_{\mathrm{A}}} B_{y}^{(i)} .
$$

This current radiates the electromagnetic field of the Alfvén wave with the components $\widetilde{B}_{y}^{(r)}$ and $\widetilde{E}_{x}^{(r)}$. The $x$-component of a total current induced is reduced to

$$
\begin{aligned}
\widetilde{J}_{x} & =\Sigma_{\mathrm{P}} \widetilde{E}_{x}^{(r)}+\Sigma_{\mathrm{H}} \widetilde{E}_{y} \\
& =\Sigma_{\mathrm{P}} \widetilde{E}_{x}^{(r)}+i \frac{2 k_{0}}{|k|(1+\operatorname{coth}|k| h)} \frac{\Sigma_{\mathrm{H}}^{2}}{\Sigma_{\mathrm{P}}+\Sigma_{\mathrm{A}}} B_{y}^{(i)} .
\end{aligned}
$$

The inductive Hall current provides the input in the amplitude of the magnetic field of the reflected Alfvén wave as follows

$$
\begin{aligned}
\widetilde{B}_{y}^{(r)}= & -\frac{4 \pi}{c} \widetilde{J}_{x}=-\frac{4 \pi}{c} \\
& \cdot\left[\Sigma_{\mathrm{P}} \widetilde{E}_{x}+i \frac{2 k_{0}}{|k|(1+\operatorname{coth}|k| h)} \frac{\Sigma_{\mathrm{H}}^{2}}{\Sigma_{\mathrm{P}}+\Sigma_{\mathrm{A}}} B_{y}^{(i)}\right] .
\end{aligned}
$$

Rewriting Eq. (A.4) with the use of $\widetilde{E}_{x}=c\left(4 \pi \Sigma_{\mathrm{A}}\right)^{-1} \widetilde{B}_{y}^{(r)}$ we obtain

$$
\widetilde{B}_{y}^{(r)}=-\frac{2 i \omega}{C_{\mathrm{A}}|k|(1+\operatorname{coth}|k| h)} \frac{\Sigma_{\mathrm{H}}^{2}}{\left(\Sigma_{\mathrm{P}}+\Sigma_{\mathrm{A}}\right)^{2}} B_{y}^{(i)} .
$$

Finally, combining Eqs. (5) and (A.5) we obtain the reflection coefficient in the first approximation

$$
R_{\mathrm{A}}=\frac{\Sigma_{\mathrm{P}}-\Sigma_{\mathrm{A}}}{\Sigma_{\mathrm{P}}+\Sigma_{\mathrm{A}}}-i \frac{\omega}{C_{\mathrm{A}}} \frac{1-\exp (-2|k| h)}{|k|} \frac{\Sigma_{\mathrm{H}}^{2}}{\left(\Sigma_{\mathrm{P}}+\Sigma_{\mathrm{A}}\right)^{2}} .
$$

Comparison of two terms in the relationship (A6) gives the condition (7). More formally, the condition (7) could be derived from the "exact" expression for $R_{\mathrm{A}}$, which can be found in Alperovich and Fedorov (1992) and Yoshikawa and Itonaga (1996).

\section{References}

Allan, W., Quarter-wave ULF pulsations, Planet. Space Sci., 31, 323-330, 1983.

Allan, W. and F. B. Knox, The effect of finite ionosphere conductivities on axisymmetric toroidal Alfvén wave resonances, Planet. Space Sci., 27, 939-950, 1979.

Alperovich, L. S. and E. N. Fedorov, On hydromagnetic wave beams propagation through the ionosphere, Ann. Geophys., 10, 647-654, 1992.

Angerami, J. J. and J. O. Thomas, The distribution of electrons and ions in the Earth's exosphere, J. Geophys. Res., 69, 4537-4560, 1964.

Baransky, L. N., A. W. Green, E. N. Fedorov, N. A. Kurneva, V. A. Pilipenko, and W. Worthington, Gradient and polarization methods of ground-based monitoring of magnetospheric plasma, J. Geomag. Geoelectr., 47, 12931309, 1995.

Bilitza, D., Solar-terrestrial models and application software, Planet. Space Sci., 40, 541-579, 1992.

Carpenter, D. L. and R. R. Anderson, An ISEE/whistler model of equatorial electron density in the magnetosphere, J. Geophys. Res., 97A, 1097-1108, 1992.

Glassmeier, K.-H., On the influence of ionospheres with non-uniform conductivity distribution on hydromagnetic waves, J. Geophys., 54, 125, 1984. 
Gugliel'mi, A. V., Coefficient of relationship between Pc 3 frequency and IMF magnitude, Geomagn. Aeron., 28, No. 3, 465, 1988.

Hameiri, E. and M. G. Kivelson, Magnetospheric waves and the atmosphereionosphere layer, J. Geophys. Res., 96A, 21125-21134, 1991.

Hughes, W. J. and D. J. Southwood, The screening of micropulsation signals by the atmosphere and ionosphere, J. Geophys. Res., 81, No. 19, 3234 3240, 1976.

Itonaga, K. and T. Kitamura, Effect of non-uniform ionospheric conductivity distributions on Pc 3-5 magnetic pulsations-Alfvén wave incidence, $J$. Geomag. Geoelectr., 40, 1413-1435, 1988.

Lyatsky, V. B. and Yu. P. Maltsev, Interaction between Magnetosphere and Ionosphere, 192pp., Nauka, Moscow, 1983 (in Russian).

Newton, R. S., D. J. Southwood, and W. J. Hughes, Damping of geomagnetic pulsations by the ionosphere, Planet. Space Sci., 26, 201-209, 1978.

Pilipenko, V. A. and E. N. Fedorov, Magnetotelluric sounding of the crust and hydromagnetic monitoring of the magnetosphere with the use of ULF waves, in Solar Wind Sources of Magnetospheric ULF Waves, edited by M. Engebretson, K. Takahashi, and M. Scholer, pp. 283-292, Geophysical Monograph, v. 81, AGU, 1994.

Pilipenko, V. A., E. N. Fedorov, N. V. Yagova, S. I. Solovyev, E. F. Vershinin, and K. Yumoto, Variations of spectral content of Pc 3-4 pulsations along geomagnetic meridian 210 ${ }^{\circ}$, Geomagn. Aeron., 37, No. 1, 80, 1997.

Pilipenko, V., K. Yumoto, E. Fedorov, N. Kurneva, and F. Menk, Field line Alfvén oscillations at low latitudes, Memoirs of Kyushu University, series D, 30, No. 1, 23-43, 1998.

Polyakov, S. V., Magnetospheric Alfvén resonance in a case of horizontallyinhomogeneous ionosphere, Geomagn. Aeron., 28, 587, 1988.

Poulter, E. M., W. Allan, and G. J. Bailey, ULF pulsation eigenperiods within the plasmasphere, Planet. Space Sci., 36, 185-196, 1988.

Saka, O., M. Itonaga, and T. Kitamura, Ionospheric control of polarization of low-latitude geomagnetic micropulsations at sunrise, J. Atmos. Terr. Phys., 44, 703-712, 1982.
Strangeways, H. J., A model for the electron temperature variation along geomagnetic fiel lines and its effect on electron density profiles and VLF paths, J. Atmos. Terr. Phys., 48, 671-683, 1986.

Vellante, M., U. Villante, M. De Lauretis, and F. Cerulli-Irelli, An analysis of micropulsation events at a low-latitude station during 1985, Planet. Space Sci., 37, No. 7, 767-773, 1989.

Vellante, M., U. Villante, M. DeLauretis, and G. Barchi, Solar-cycle variation of the dominant frequencies of Pc 3 geomagnetic pulsations at $L=1.6$, Geophys. Res. Lett., 23, 1505-1508, 1996.

Waters, C. L., F. V. Menk, and B. J. Fraser, The resonance structure of low latitude Pc 3 geomagnetic pulsations, Geophys. Res. Lett., 18, 2293-2296, 1991.

Yoshikawa, A. and M. Itonaga, Reflection of shear Alfvén waves at the ionosphere and the divergent Hall current, Geophys. Res. Lett., 23, 101104, 1996.

Yumoto, K. and $210^{\circ} \mathrm{MM}$ Observation Group, Globally coordinated magnetic observations along $210^{\circ}$ magnetic meridian during STEP period, $J$. Geomag. Geoelectr., 44, 261-276, 1992.

Yumoto, K., V. Pilipenko, E. Fedorov, N. Kurneva, and K. Shiokawa, The mechanisms of damping of geomagnetic pulsations, J. Geomag. Geoelectr., 47, 163-176, 1995.

Yumoto, K. and the $210^{\circ} \mathrm{MM}$ Magnetic Observation Group, The STEP $210^{\circ}$ magnetic meridian network project, J. Geomag. Geoelectr., 48, 12971309, 1996.

Ziesolleck, C. W. S., B. J. Fraser, F. W. Menk, and P. W. McNabb, Spatial characteristics of low-latitude Pc 3-4 geomagnetic pulsations, J. Geophys. Res., 98A, 197-207, 1993.

N. Yagova, V. Pilipenko (e-mail: vpilipenko@uipe-ras.ru), E. Fedorov, $\mathrm{M}$. Vellante, and K. Yumoto 